\title{
El consumo de alcohol disminuye el tiempo de lactancia exclusiva
}

\author{
MARIO MORAES ${ }^{1}$, PATRICIA AMERIO ${ }^{2}$, ROCÍO VALIERO ${ }^{2}$, CLAUDIO SOSA $^{3}$ \\ 1. Profesor Adj. Encargado Curso Neonatología, Escuela de Parteras, UDELAR. Prof. Adj. Dpto de Neonatología CHPR, UDELAR. \\ 2. Parteras Obstetras, Escuela de Parteras, UDELAR. \\ 3. Prof. Adj. Clínica Ginecológica C, CHPR, UDELAR.
}

\begin{abstract}
Alcohol ingestion and maternal breast feeding

Objectives: The aim of this study was to determine factors associated with exclusive breastfeeding in the first month of life. Method: We conducted a cohort study with prospective data acquisition. In the first 48 hours of life during hospitalization were collected known factors, beliefs and attitudes that are associated with breastfeeding. Telephone follow-up was performed at month of life. Results: We included 273 mothers and children housed in a room together. The loss was 11 (4\%). In 191 mothers who were contacted in a month and had one or more child before, $49(77.2 \%)$ had breastfeed their children 6 months or more. 116 mothers $(42.6 \%)$ received information on the benefits of breastfeeding during pregnancy. 140 mothers $(51.4 \%)$ reported pain during breastfeeding, 74 of them with cracks. 148 infants $(54.4 \%)$ latched in the first hour of life. 78 (28.6\%) received bottle by neonatal indication or maternal difficult. $149(56.8 \%)$ continued with exclusively breastfed in a month. Alcohol consumption significantly reduced the possibility of exclusive breastfeeding in a month $(p=0.01)$. In patients with a previous child was significantly associated with exclusive breastfeeding in a month have given the chest more than six months previously $(\mathrm{p}=0.001)$. Remain valid after multivariate analysis statistical history of breastfeeding more than 6 months OR 2.91 (95\% CI 1.54 to 5.50), alcohol OR 0.55 (95\% CI 0.32 to 0.92 ). Conclusions: Alcohol consumption was independently associated as a negative predictor for exclusive breastfeeding. Having given breast more than six months before and be multiparous increases the possibility of exclusively breastfeeding in a month.
\end{abstract}

(Key words: Breast feeding, alcohol drinking, risk factors, infant, newborn).

Arch Pediatr Urug 2010; 81(1): 16-22

\section{RESUMEN}

Objetivos: El objetivo de este estudio es determinar qué factores se asocian con la lactancia exclusiva al mes de vida. Método: Se realizó un estudio de tipo cohorte con adquisición prospectiva de los datos. En las primeras 48 horas de vida durante la internación se recabaron factores conocidos, creencias y actitudes que se asocian con lactancia materna. Se realizó el seguimiento telefónico al mes de vida. Resultados: Se incluyeron 273 madres con sus hijos, alojados en habitación conjunta. La pérdida fue de 11 (4\%). De las 191 madres

Correspondencia a:

Mario Moraes

E-mail: marmorae@gmail.com 
que se contactaron al mes y tenían una para o más amamantaron anteriormente a sus hijos más de 6 meses 149 (77,2\%). Recibieron información sobre los beneficios de la lactancia durante el embarazo 116 madres $(42,6 \%)$. Refieren dolor al amamantar $140(51,4 \%)$ madres de las cuales 74 presentan grietas. Se prendieron al pecho en la primera hora de vida $148(54,4 \%)$ recién nacidos. Recibieron biberón 78 (28,6\%) por indicación neonatal o por dificultad materna. Siguieron con pecho exclusivo al mes $149(56,8 \%)$. El consumo de alcohol redujo en forma significativa la posibilidad de dar pecho exclusivo al mes $(p=0,01)$. En las pacientes con un hijo previo se asoció en forma significativa a lactancia exclusiva al mes el haber dado pecho más de 6 meses con anterioridad $(\mathrm{p}=0,001)$. Mantienen su validez estadística luego del análisis multivariado antecedente de lactancia más de 6 meses OR 2,91 (IC95\% 1,54-5,50), consumir alcohol OR 0,55 (IC95\% 0,32-0,92). Conclusiones: se asoció en forma independiente como predictor negativo para lactancia exclusiva el consumo de alcohol. Haber dado pecho por más de 6 meses anteriormente y ser multípara aumenta la posibilidad de lactar en forma exclusiva al mes.

(Palabras clave: Lactancia materna, consumo de bebidas alcohólicas, factores de riesgo, recién nacido). Arch Pediatr Urug 2010; 81(1): 16-22

\section{ESTE TRABAJO LO PUEDE ENCONTRAR EN EXTENSO EN WWW.SciELO.ORG}

